\title{
A review of electroencephalographic changes in diabetes mellitus in relation to major depressive disorder
}

This article was published in the following Dove Press journal:

Neuropsychiatric Disease and Treatment

16 January 2013

Number of times this article has been viewed

\author{
Anusha Baskaran ${ }^{1,2}$ \\ Roumen Milev ${ }^{3}$ \\ Roger S Mclntyre ${ }^{2}$ \\ 'Centre for Neuroscience Studies, \\ Queen's University, Kingston; ${ }^{2}$ Mood \\ Disorders Psychopharmacology Unit, \\ University Health Network, Toronto; \\ ${ }^{3}$ Department of Psychiatry, Queen's \\ University, Kingston, Canada
}

\begin{abstract}
A bidirectional relationship exists between diabetes mellitus (DM) and major depressive disorder (MDD), with depression commonly reported in both type 1 DM (T1DM) and type $2 \mathrm{DM}$ (T2DM), and depressive symptoms associated with a higher incidence of diabetes. However, how the two conditions are pathologically connected is not completely understood. Similar neurophysiological abnormalities have been reported in both DM and MDD, including elevated electroencephalographic (EEG) activity in low-frequency slow waves and increased latency and/or reduced amplitude of event-related potentials. It is possible that this association reflects some common underlying pathology, and it has been proposed that diabetes may place patients at risk for depression through a biological mechanism linking the metabolic changes of DM to changes in the central nervous system. In this review we will discuss EEG abnormalities in DM, as well as the biological mechanisms underlying various EEG parameters, in order to evaluate whether or not a common EEG biosignature exists between DM and MDD. Identifying such commonalities could significantly inform the current understanding of the mechanisms that subserve the development of the two conditions. Moreover, this new insight may provide the basis for informing new drug discovery capable of mitigating and possibly even preventing both conditions.
\end{abstract}

Keywords: electroencephalography, event-related potential, diabetes mellitus, major depressive disorder

\section{Introduction}

Diabetes mellitus (DM) remains a global epidemic, with an estimated 285 million people affected by diabetes worldwide. ${ }^{1}$ There are two major types of DM: insulindependent diabetes (type 1 DM [T1DM]) and noninsulin-dependent diabetes (type 2 DM [T2DM]). T1DM, also known as juvenile diabetes, occurs when the immune system attacks and destroys the cells of the pancreas that produce insulin. In T2DM, individuals are still able to produce insulin, but their bodies do not respond to it normally (ie, insulin resistance). Approximately $10 \%$ of people with diabetes have T1DM, whereas the remainder have T2DM. The rising prevalence of DM is a consequence of the significant increase in the latter type. ${ }^{1}$

DM possesses a bidirectional relationship with major depressive disorder (MDD). ${ }^{2}$ MDD is common in T1/T2DM and has significant effects on the course and outcome of diabetes. ${ }^{3}$ Some studies have shown that the risk for depression is particularly elevated in T1DM, ${ }^{4}$ whereas others suggest a specific association with T2DM. ${ }^{5}$ Likewise, elevated depressive symptoms have been associated with a higher risk of developing T2DM. ${ }^{6}$ In a recent population-based study, Chien et $\mathrm{al}^{7}$ reported the 1 -year prevalence
Correspondence: Anusha Baskaran 399 Bathurst Street, Toronto, Ontario, M5T 2S8, Canada

Tel +I(4I6) 6035800 ext. 2024

$\mathrm{Fax}+\mathrm{I}(416) 6035368$

Email Anusha.Baskaran@uhn.ca 
of DM in patients with MDD to be higher than that of the general population $(11.65 \%$ vs $6.53 \%$; odds ratio $1.53 ; 95 \%$ confidence interval 1.39-1.69). Comorbid MDD in people with diabetes and vice versa forms a serious threat to quality of life and increases the risk for the development of cardiovascular complications of DM, leading to increased mortality rates and higher health care costs. ${ }^{5}$ However, how the two conditions are connected remains insufficiently characterized. Identifying a common underlying mechanism between DM and MDD could greatly aid in a better understanding toward the prevention and treatment of patients who are comorbid for both conditions. Indeed, enhanced efforts toward good glycemic control have been shown to contribute to improvements in mood and perceptions of well-being. ${ }^{3}$ Also, improving depressive symptoms has been shown to improve or even alleviate chronic illnesses such as DM. ${ }^{8}$ An integrated understanding of the mechanisms that subserve both DM and MDD will need to take into consideration accumulated evidence implicating central nervous system (CNS) abnormalities associated with both conditions.

One mechanism that has been used widely to investigate CNS alterations in DM is electroencephalography (EEG). EEG is a measure of the brain's spontaneous electrical activity acquired from many different electrodes placed on the scalp. Recorded activity at each electrode is the gross measure of electrical activity arising from a number of different neurons in cortical areas surrounding each electrode. EEG signals are described in terms of either absolute or relative power. Absolute power is the amount of power in an EEG frequency band at a given electrode measured in microvolts $\left(\mathrm{uV}^{2}\right)$. Relative power is the percentage of power in any frequency band compared with the total power of the entire EEG spectrum. A more advanced form of EEG, called quantitative EEG (QEEG), now exists. In QEEG, electrical signals from the brain are converted to digital form, which allows patterns undetectable by the naked eye to be revealed.

Diabetes may place patients at risk for depression through a biological mechanism linking the metabolic changes of DM to changes in the CNS. ${ }^{9}$ Keeping with this perspective, McIntyre et $a l^{10}$ demonstrated that a common point of pathophysiology exists between MDD and DM when considering brain volume abnormalities and neurocognitive deficits. In this review, we discuss the literature pertinent to EEG abnormalities in DM, but we also discuss biological mechanisms underlying each EEG parameter discussed, in order to evaluate whether or not a common link exists between neurobiological mechanisms associated with EEG findings in DM and in MDD.

\section{EEG frequency band activity}

Spectral power in four main EEG frequency bands has been explored in DM, including activity in the delta $(<4 \mathrm{~Hz})$, theta (4-8 Hz), alpha (8-13 Hz), and beta $(13-30 \mathrm{~Hz})$ bands. Delta waves accompany slow-wave sleep, theta waves reflect a state of drowsiness, and alpha and beta waves generally reflect a relaxed state and an active brain, respectively. Gamma (31-44 Hz) waves have also been investigated but with less focus when compared with the other EEG bands. The normal EEG spectrum is dominated by alpha and beta waves, whereas theta waves are a minor component and delta waves are normally absent. Alpha, beta, and gamma make up the high-frequency fast waves, whereas the low-frequency slow waves comprise delta and theta.

\section{High-frequency fast-wave activity}

EEG studies of both adults and children with diabetes generally reveal a decrease in high-frequency fast-wave activity. When reports of EEG findings in children were scarce, EegOlofsson and Petersen ${ }^{11}$ reported a significantly lower level of alpha activity in diabetic children and adolescents with DM ( $n=69$, mean age 12.8 years). Pathological EEGs in this study were shown to significantly correlate with hypoglycemia. Similar results were demonstrated in a follow-up study. ${ }^{12}$ Topographic EEG mapping in a group of young patients with T1DM ( $=14$; eight men, six women; $33.1 \pm 8.9$ years) in later years also revealed decreased alpha activity $(P<0.05)$ during induced hypoglycemia. ${ }^{13}$ In contrast to these reports, Bjørgaas et $\mathrm{al}^{14}$ found a moderate increase in alpha band activity over fronto-central and temporal regions during controlled hypoglycemia in 19 diabetic children (mean age $14.2 \pm 1.4$ years) using QEEG.

One early study demonstrated that adults with T1DM have a reduction in relative alpha power in frontal and central areas, with no difference in the incidence of hypoglycemia between patients with normal and pathological EEGs. ${ }^{15}$ However, biochemically induced hypoglycemia in adults with T1DM ( $n=13$, median age 28 years [range $20-40$ years]) has been shown to decrease alpha activity in the parieto-occipital and temporal regions. ${ }^{16}$ In a similar study, high-frequency alpha activity $(9.75-12.87 \mathrm{~Hz})$ was reduced over posterior regions of the brain, whereas low-frequency alpha activity $(8.12-9.62 \mathrm{~Hz})$ was elevated over the anterior brain. ${ }^{17}$

Elderly patients with T2DM ( $n=43$ males) have been reported to have slowing of EEG activity over the central cortex and reduction in alpha activity over the parietal area, compared with age-matched nondiabetics $\left(\mathrm{n}=41\right.$ males). ${ }^{18}$ Adolescents with T1DM and previous ketoacidotic episodes 
$(n=44)$ have been found to have decreased relative alpha power, suggesting a role for hyperglycemia. ${ }^{19}$ This finding is in direct contrast to the largely supported role of low blood glucose level in EEG abnormalities observed in DM. A similar study showed pronounced loss of alpha oscillations in posterior temporal regions $(P<0.05$ or 0.01$)$, along with the alpha peak frequency in temporo-central regions being reduced bilaterally $(P<0.01)$ in T1DM patients $(\mathrm{n}=49)$ compared with controls $(n=51) .{ }^{20}$ However, both of these changes were found not to correlate with a previous history of hypoglycemia. Two recent papers investigating EEG change in T1DM patients confirmed reports from earlier EEG studies by demonstrating a global reduction of alpha band activity $(P<0.04)$ with the most pronounced regional difference over posterior temporal regions $(P<0.001) .{ }^{21,22}$ Again, this reduction was found not to correlate with hypoglycemia. ${ }^{21}$

Reduced activity in the beta band has also been reported in people with diabetes. Elderly patients with T2DM demonstrate decreased beta activity over posterior regions of the brain. ${ }^{18}$ In contrast, diabetic children have been reported to have elevated beta band activity over fronto-central and temporal regions. ${ }^{14}$ Studies conducted in more recent years support the earlier observation of reduced beta activity in DM. T1DM patients with hypoglycemia $(\mathrm{N}=13)$ have been shown to have reduced absolute power in the beta band (13-35 Hz) and slowing of centroid frequencies of beta and total frequency bands $(1.3-35 \mathrm{~Hz}$ ) (up to $P<0.01)$. ${ }^{23}$ In two later studies of young adults with T1DM, reports of decreased beta band activity were not found to correlate with a previous history of hypoglycemia..$^{20,21}$ One of these studies reported reduced activity in the beta band bilaterally in the anterior temporal and occipital regions (T3, T4, O1, and $\mathrm{O} 2, P<0.05$ or 0.01$),{ }^{20}$ whereas the latter reported a reduction over bilateral posterior temporal regions $(P<0.01) .{ }^{21}$ Reduced power in the beta band over central and posterior temporal regions was also demonstrated in very recent studies of individuals with TI/T2DM. ${ }^{22,24}$ EEG power did not correlate with metabolic control. ${ }^{22}$ Instead, a positive correlation was found between verbal ability and resting EEG power in the alpha $(P<0.03)$ and beta $(P<0.04)$ bands. $^{22}$

Only a handful of EEG studies have reported reduced gamma activity in DM patients. Young adults with T1DM have been shown to have decreased gamma oscillations bilaterally over posterior temporal regions $(P<0.05$ or 0.01$) .{ }^{20,21}$ More recently, Cooray et $\mathrm{al}^{22}$ showed global reduction of gamma band activity $(P<0.005)$ in T1DM patients, which was most pronounced over mid-parietal regions $(\mathrm{Pz}, P<0.001)$ and was found to negatively correlate with visual-spatial ability
$(P<0.04)$. No correlation to hypoglycemia was reported in all three studies mentioned. ${ }^{20-22}$

\section{Low-frequency slow-wave activity}

EEG studies of diabetic patients generally report an increase in low-frequency slow-wave activity. One early report demonstrated enlarged slow waves in the left parietal area of adults with T1DM. ${ }^{15}$ Similarly, Eeg-Olofsson and Petersen ${ }^{11}$ reported increased theta activity in diabetic children $(n=69$, mean age 12.8 years), which correlated with hypoglycemic conditions. Pathological activity within theta and/or delta bands has also been demonstrated in young individuals with uncontrolled T1DM who were newly diagnosed with ketoacidosis or ketosis $(n=39$, age 11 months to 16 years $) .^{25}$ This finding supports a probable role for hyperglycemia, which was in contrast to the widely reported connection between EEG abnormalities in DM and hypoglycemia.

Elderly patients with T2DM have been shown to have slowing of EEG with significantly greater power in the theta band at central midline areas $(P<0.05) .{ }^{18}$ Elevated theta activity in temporal and parieto-occipital areas has been demonstrated in adults with T1DM $(n=13)$ during induced hypoglycemia. ${ }^{16}$ In a similar study, theta activity was shown to increase over anterior regions, whereas delta activity increased over posterior regions. ${ }^{17}$ Adolescents with T1DM also demonstrate increased theta and delta activity, which has been shown to be related to poor metabolic control and ketoacidosis. ${ }^{19}$ Tribl et al ${ }^{13}$ reported similar results over lateral frontal regions, in the range of slight hypoglycemia. However, during deep hypoglycemia, there was a topographic maximum of slow frequencies in posterior regions of the brain, particularly from the centro-temporal region to the parieto-occipital region. ${ }^{13}$

Diabetic children $(n=19$, mean age $14.2 \pm 1.4$ years $)$ have been shown to have marked and widespread increase in low-frequency activity during controlled hypoglycemia with the increase in theta activity observed globally in all cortical areas, whereas the increase in delta activity was mainly due to an $11 \%$ increase in the temporal region $(P<0.0005) .{ }^{14}$ A small but significant increase in theta and delta amplitudes was also observed but with no regional differences. ${ }^{14}$ In this particular study, the increase in slow-wave activity correlated with hypoglycemic symptom score. ${ }^{14}$ Young adults with well-controlled T1DM without a history of recurrent hypoglycemia also revealed elevated delta activity in frontal (F4-F8) and temporo-parietal (T5-P3) regions, and increased theta activity in frontal and left central positions (F3, Fz, F8, and C3, $P<0.05) .{ }^{20}$ Hyllienmark et al ${ }^{21}$ found 
similar results in adolescents with T1DM, where a history of severe hypoglycemia correlated with a global increase in theta activity, whereas poor metabolic control correlated with elevated delta activity. In contrast to these previous findings, one recent study found a global reduction in the power of slow-wave activity $(P<0.01)$ in T1DM patients, with a positive correlation found between verbal ability and resting EEG power in the theta band $(P<0.04){ }^{22}$

\section{EEG: neurobiological mechanism and relation to depression}

While earlier research attributed EEG abnormalities to reduced blood glucose in diabetics, more recent studies have generally failed to find strong support of such a view. Rather, recent studies have demonstrated a role for cognitive processes such as visual-spatial ability and verbal ability. ${ }^{20,22,24}$

According to the "metabolic hypothesis," metabolic defect in DM may lead to permanent cerebral dysfunction. ${ }^{12}$ However, even in early reports of EEG abnormalities in diabetic patients, authors stressed the fact that although repeated episodes of hypoglycemia may cause functional and metabolic disturbances in neurons, they are still probably of minor importance as causal factors, because diabetic patients with no sign of hypoglycemia also have EEG abnormalities. ${ }^{11,12}$ In one early study of diabetic patients, the incidence of hypoglycemia seemed to be as frequent in patients with a normal EEG as in those with a pathological EEG. ${ }^{15}$ Moreover, minor hypoglycemic episodes have been shown to have no effect on EEG. ${ }^{26}$ Interestingly, abnormal EEG power spectra have been demonstrated in diabetic patients with well-controlled T1DM without history of recurrent hypoglycemia. ${ }^{20}$ Hence, studies of diabetic patients in the literature do not always clearly relate abnormal EEG findings with blood glucose level. This may be due to the fact that there are factors beyond the previously supported "metabolic hypothesis." With fewer and fewer studies supporting the role of hypoglycemia in EEG abnormalities in DM, cognitive processes have been recently brought to the forefront. The EEG abnormalities shared between DM and MDD may aid in better understanding this relationship.

Similar alterations of EEG frequency bands as those observed in DM have been demonstrated in MDD patients, namely elevated slow-wave activity (see Table 1). Lowfrequency EEG activity including theta and delta power has been shown to be increased over the right hemisphere in depressed patients $(n=20$, mean age $44.31 \pm 9.31$ years $)$ compared with age-matched healthy controls $(n=20$, mean age $44.15 \pm 9.22$ years). ${ }^{27}$ Korb et $\mathrm{al}^{28}$ used low-resolution electromagnetic tomography analysis and found elevated delta and theta power in the anterior cingulate cortex (ACC) of depressed patients, a region of the brain shown to be dysfunctional in MDD. Pizzagalli et $\mathrm{al}^{29}$ reported that the rostral ACC produces the largest cluster of positive correlations between theta current density and glucose metabolism. Positive correlations were also found in right fronto-temporal regions, revealing a connection between theta band activity and cerebral metabolism in the ACC as well as the disruption of functional connectivity within fronto-cingulate pathways in depression. ${ }^{29}$ More recently, elevated theta activity has also been found in parietal and occipital electrode sites from EEG recordings of depressed patients versus nondepressed subjects. $^{30}$

Dysfunctional cognitive processes have been documented in MDD in relation to EEG alterations. ${ }^{31}$ Theta activity is viewed as an EEG index of activity in the default mode network ${ }^{32}$ in which the rostral ACC is a main hub with involvement in self-focused processing. ${ }^{33}$ Elevated resting state activity in the default mode network is associated with focusing on reflective thought or task-independent introspection such as rumination, remembering, and planning. ${ }^{34}$ Rumination, in particular, has

Table I Directional EEG alterations in DM and MDD and their associated brain regions

\begin{tabular}{lllll}
\hline EEG parameter & TIDM & T2DM & MDD & Brain region \\
\hline Resting EEG & & & & Posterior temporal \\
Alpha band power & $\downarrow$ & $\downarrow$ & $\uparrow$ & Temporal \\
Beta band power & $\downarrow$ & $\downarrow$ & $\uparrow$ & Posterior temporal \\
Gamma band power & $\downarrow$ & $\uparrow$ & $\uparrow$ & Frontal and parieto-occipital; ACC \\
Theta band power & $\uparrow$ & $\uparrow$ & $\uparrow$ & Frontal and temporo-parietal; ACC \\
Delta band power & $\uparrow$ & $\uparrow$ & $\uparrow$ & Posterior \\
Event-related potentials & $\uparrow$ & $/$ & $\downarrow$ & Frontal and temporo-parietal \\
P300 latency & $\downarrow$ & $\downarrow$ & $\downarrow$ & Central and posterior \\
P300 amplitude & $\downarrow$ & & & \\
NI00 amplitude & & & $\uparrow$ & \\
\hline
\end{tabular}

Abbreviations: ACC, anterior cingulate cortex; DM, diabetes mellitus; EEG, electroencephalographic; MDD, major depressive disorder; TIDM, type I diabetes mellitus; T2DM, type 2 diabetes mellitus. 
been shown to play a role in affective distress in people with chronic physical illnesses such as diabetes. ${ }^{8}$ Moreover, slowwave activity in DM has also been shown to be reflective of cognitive mechanisms such as verbal activity. ${ }^{22}$ Hence, not only may cognitive dysfunction serve as a better explanation toward low-frequency EEG abnormalities observed in DM but it may also represent a common biosignature between DM and MDD.

Although a focal decrease in fast-wave activity has been largely reported in the posterior temporal area of diabetics, MDD has been shown to be associated with elevated activity in these EEG bands (see Table 1). Singer ${ }^{35}$ found high-frequency oscillations in the beta and gamma bands in depressed subjects, in a state of focused attention. Gamma band activity has also been closely related to visual information processing and attentional perceptual mechanisms. ${ }^{36}$ In DM, reduced gamma band activity has been associated with visual spatial ability. ${ }^{22}$ However, it is unlikely that DM and MDD share a common neurobiological mechanism for altered fast-wave activity, due to the reported difference of directionality in high-frequency EEG band activity (see Table 1).

\section{Event-related potentials}

Different from spontaneous EEG, evoked potentials represent electrical potentials recorded from the CNS following the presentation of a stimulus. Event-related potentials (ERPs) are a measure of change in voltage, which reflect the brain's transient electrical response to stimuli. ERPs are quantified in terms of components that comprise distinctive peaks and troughs reflective of positive and negative fluctuations in voltage ( $\mathrm{P}$ for positive and $\mathrm{N}$ for negative). Components are identified and named based on the polarity of the waveform deflection and on the latency at which the waveform occurs post stimulus. Two ERP components that have been the main focus of investigation in DM are P300 and N100.

\section{The P300 component}

The P300 component is a positive component measured at approximately $300 \mathrm{msec}$ after the presentation of taskrelevant (target) stimuli. This ERP index is elicited through the superposition of the delta and theta band oscillatory responses and is believed to reflect the cognitive processes of attention and auditory processing. ${ }^{37,38}$

Patients with T1DM have been shown to have longer P300 latencies. ${ }^{17,39-43}$ However, no difference in P300 latency has been found between T1DM patients with and without history of hypoglycemic coma. ${ }^{40}$ Prolonged P300 latency observed by Tallroth et $\mathrm{al}^{17}$ in T1DM diabetics $(\mathrm{n}=8$, mean age $28.0 \pm 7.4$ years) versus healthy controls $(n=12$, mean age $26.4 \pm 4.2$ years) was found to be correlated to diabetes duration but not to total hypoglycemic episodes. This group also reported reduction of the P300 amplitude, which was found to be associated with hypoglycemia, but did not restore after normalization of blood glucose levels. ${ }^{17}$

Significant reduction of the P300 amplitude has been demonstrated in T1DM patients with poor metabolic control, whereas no such reduction was observed in patients with strict control. ${ }^{41}$ In a more recent study of T1DM patients $(n=119)$ versus healthy controls $(n=61)$, elevated P300 latency correlated with low cognitive global score, whereas a slight decrease in the P300 amplitude correlated with psychomotor speed. ${ }^{39}$

Studies have also demonstrated increased P300 latency in T2DM patients, with the largest difference between diabetic patients and healthy controls observed in the posterior region of the brain. ${ }^{18,24,44-46}$ Blood glucose level has been shown not to correlate with reduced P300 latency in T2DM. ${ }^{46}$

\section{The NI00 component}

The N100 component, measured $100 \mathrm{msec}$ after presentation of an auditory stimulus, is elicited by physical characteristics of a stimulus in the absence of task demands. It is thought to reflect processes of the primary and association auditory cortices. $^{47}$

One early study revealed reduced amplitude in the N100 component in patients with T1DM $(n=50) .{ }^{48}$ More recently, Cooray et $\mathrm{al}^{39}$ found normal N100 latency but a highly significant decrease in the N100 amplitude in patients with T1DM. This finding correlated with a decrease in psychomotor speed but not with function in other cognitive domains. ${ }^{39}$ Patients with T2DM have also been shown to have lower N100 amplitude when compared with healthy control subjects, mainly over the central and posterior regions of the brain. ${ }^{24}$

\section{ERP: neurobiological mechanism and relation to depression}

Similar to the literature on EEG frequency band abnormalities in DM, the majority of ERP studies in diabetes do not lend support to the role of blood glucose level in observed abnormalities. The prolonged P300 latency observed in T1DM has been found not to correlate with hypoglycemic episodes. ${ }^{17}$ Moreover, no difference in P300 latencies has been found between T1DM patients with and without history of hypoglycemic coma. ${ }^{40}$ Although reduced P300 amplitude has been found to be associated with hypoglycemia in T1DM, normalization of blood glucose levels has failed to restore the reduction. ${ }^{17}$ Also, Tandon et $\mathrm{al}^{46}$ demonstrated that P300 
latency or amplitude in T2DM does not show any correlation with blood glucose level. Hence, like EEG studies, ERP studies of diabetic patients do not support a clear relation between abnormal ERP findings and blood glucose level.

ERP abnormalities are not specific to DM, because similar findings have been reported in association with MDD (see Table 1). In depressed patients, a delay in the latency of the P300 component has been demonstrated over the bilateral temporal lobes, the left frontal region, and the right temporal parietal area. ${ }^{49-53}$ Early ERP studies in MDD have also reported a reduction of the P300 amplitude. ${ }^{54}$ More recent studies have reported similar findings. ${ }^{49,51,52,55-57}$

According to Sara et al, ${ }^{54}$ ERPs can be divided into early $(<100 \mathrm{~ms})$ and late $(>100 \mathrm{~ms})$ occurring components. Early ERP components such as N100 are thought to reflect the activity in sensory nerves, brainstem, thalamus, and primary sensory cortex. ${ }^{58}$ Late components such as P300 reflect aspects of information processing such as attention, allocation, and decision making. ${ }^{59}$ It has been suggested that P300 is elicited at the end of a cognitive episode at which time, a decision is made as to whether a stimulus is important or not. ${ }^{60}$ According to this perspective, P300 latency may reflect the discrimination time needed to interpret a stimulus, whereas the P300 amplitude has been correlated with psychological variables such as expectancy, attention, and meaning of the stimulus. ${ }^{53}$ In addition, the P300 component is also thought to index the updating of neurocognitive models that are concerned with the prediction of future events. ${ }^{54,61}$ Cognitive theories of depression have proposed that depression may be characterized by abnormalities in the ability to predict future events. ${ }^{62,63}$ In DM, both prolonged P300 latency and reduced amplitude have also been linked with cognition. ${ }^{39}$ Whereas P300 latency has been shown only in relation to a decline in global cognitive score in diabetic patients, the P300 amplitude has been particularly linked with psychomotor speed. Psychomotor speed is defined by the amount of time it takes an individual to process a signal, prepare a response, and execute that response. Therefore, the P300 component may provide a common neurophysiological index of cognitive dysfunction between DM and MDD.

Depressed patients have been shown to have increased N100 latency, ${ }^{52}$ with some studies reporting reduced N100 amplitude $^{64-66}$ and others reporting an increase. ${ }^{51}$ Burkhart and Thomas ${ }^{64}$ suggested that the significant reduction they observed in the N100 peak amplitude at the frontal scalp location between depressed patients and controls was reflec- tive of differences in arousal level or sensory sensitivity. The reason for this is that the $\mathrm{N} 100$ component responds mainly to physical characteristics of a stimulus and is thought to reflect initial extraction of information from sensory analysis of the presented stimulus. ${ }^{67,68}$ The increase in the N100 amplitude, as reported by Kemp et al, ${ }^{51}$ is interpreted as greater sensory sensitivity, ${ }^{64,67}$ which is thought to reflect heightened initial cortical innervation. ${ }^{69}$ Sources relating to the N100 component appear to include the supra temporal primary auditory cortex and direct thalamo-cortical projections to frontal and parietal areas. ${ }^{68,70,71}$ In DM, reduced N100 amplitude has also been associated with cognitive processes such as psychomotor speed. ${ }^{24,48}$ However, due to the lack of similarities in the findings arising from ERP studies investigating N100 in DM and MDD (see Table 1), the neurobiological mechanisms mediating this ERP index are most likely not congruent in the two conditions.

\section{Conclusion}

Similar neurophysiological abnormalities have been reported in both DM and MDD, including elevated EEG activity in low-frequency slow waves and increased latency and reduced amplitude of the P300 ERP component (see Table 1). EEG studies in DM have failed to find consistent support toward the role of low blood glucose level in frequency band alterations and ERP abnormalities. Hence, the origin of neurophysiological disruptions in DM may not exclusively be due to metabolic disturbances. Rather, cognitive dysfunction may govern such abnormalities in DM, as this is the case in MDD. Keeping with this perspective, disruptions in cognitive processes have been reported in both conditions, in association with EEG abnormalities. This leads to the notion that DM and MDD may share common neurobiological mechanisms that underlie EEG alterations. Taken together, DM and MDD can be conceptualized as multisystem disorders, with the CNS as an integral component. Although most studies have evaluated neurophysiological changes as a measure of CNS abnormalities in DM or MDD alone, future studies are warranted to examine the two conditions together in order to identify common neurobiological mechanisms governing CNS dysfunction. Such studies could play a useful role in better understanding mechanisms that commonly underlie the development of DM and MDD by refining biosignatures that may inform new drug discovery in the treatment of both conditions.

\section{Disclosure}

Roger S McIntyre is on speaker/advisory boards for, or has received research funds from, AstraZeneca, Bristol-Myers 
Squibb, France Foundation, GlaxoSmithKline, Eli Lilly, Janssen-Ortho, Organon, Lundbeck, Pfizer, National Alliance for Research on Schizophrenia and Depression, National Institutes of Mental Health, Stanley Medical Research Institute, Shire, and Merck. Roumen Milev is on speaker/ advisory boards for, or has received research funds from, AstraZeneca, Biovail, BrainCells Inc, Bristol-Myers Squibb, Canadian Network for Mood and Anxiety Treatments, Eli Lilly, Janssen-Ortho, Lundbeck, Pfizer, Servier, Takeda, Wyeth, and Merck. Anusha Baskaran declares no conflict of interest.

\section{References}

1. International Diabetes Federation: IDF Diabetes Atlas, 4 th ed. Brussels, Belgium: International Diabetes Federation; 2009.

2. Pan A, Lucas M, Sun Q, et al. Bidirectional association between depression and type 2 diabetes mellitus in women. Arch Intern Med. 2010; 170(21):1884-1891.

3. Lustman PJ, Clouse RE. Depression in diabetic patients: the relationship between mood and glycemic control. J Diabetes Complications. 2005; 19(2):113-122.

4. Jacobson AM, Samson JA, Weinger K, Ryan CM. Diabetes, the brain, and behavior: is there a biological mechanism underlying the association between diabetes and depression? Int Rev Neurobiol. 2002;51: 455-479.

5. Nouwen A, Nefs G, Caramlau I, et al. Prevalence of depression in individuals with impaired glucose metabolism or undiagnosed diabetes: a systematic review and meta-analysis of the European Depression in Diabetes (EDID) Research Consortium. Diabetes Care. 2011;34(3): 752-762.

6. Demakakos P, Pierce MB, Hardy R. Depressive symptoms and risk of type 2 diabetes in a national sample of middle-aged and older adults: the English longitudinal study of aging. Diabetes Care. 2010;33(4): 792-797.

7. Chien IC, $\mathrm{Wu}$ EL, Lin CH, et al. Prevalence of diabetes in patients with major depressive disorder: a population-based study. Compr Psychiatry. 2012;53(5):569-575.

8. Soo H, Burney S, Basten C. The role of rumination in affective distress in people with a chronic physical illness: a review of the literature and theoretical formulation. J Health Psychol. 2009;14(7):956-966.

9. McIntyre RS, Soczynska JK, Konarski JZ, et al. Should depressive syndromes be reclassified as "metabolic syndrome type II"? Ann Clin Psychiatry. 2007;19(4):257-264.

10. McIntyre RS, Kenna HA, Nguyen HT, et al. Brain volume abnormalities and neurocognitive deficits in diabetes mellitus: points of pathophysiological commonality with mood disorders? Adv Ther. 2010;27(2): 63-80.

11. Eeg-Olofsson O, Petersen I. Childhood diabetic neuropathy: a clinical and neurophysiological study. Acta Paediatr Scand Suppl. 1966;55: 163-176.

12. Eeg-Olofsson O. Hypoglycemia and neurological disturbances in children with diabetes mellitus. Acta Paediatr Scand Suppl. 1977;270: 91-96.

13. Tribl G, Howorka K, Heger G, Anderer P, Thoma H, Zeitlhofer J. EEG topography during insulin-induced hypoglycemia in patients with insulin-dependent diabetes mellitus. Eur Neurol. 1996;36(5):303-309.

14. Bjørgaas M, Sand T, Vik T, Jorde R. Quantitative EEG during controlled hypoglycemia in diabetic and non-diabetic children. Diabet Med. 1998;15:30-37.

15. Herlin KM, Karlsson B, Sterky G. EEG studies pm cases of juvenile diabetes mellitus of long duration. Acta Paediatrica. 1962;51(S130): $57-63$.
16. Pramming S, Thorsteinsson B, Stigsby B, Binder C. Glycaemic threshold for changes in electroencephalograms during hypoglycaemia in patients with insulin dependent diabetes. BMJ. 1988;296:665-667.

17. Tallroth G, Lindgren M, Stenberg G, Rosen I, Agardh CD. Neurophysiological changes during insulin-induced hypoglycemia and in the recovery period following glucose infusion in type 1 (insulindependent) diabetes mellitus and in normal man. Diabetologia. 1990;33: 319-323.

18. Mooradian AD, Perryman K, Fitten J, Kavonian GD, Morley JE. Cortical function in elderly non-insulin dependent diabetic patients. Behavioral and electrophysiologic studies. Arch Intern Med. 1988;148: 2369-2372.

19. Hauser E, Strohmayer C, Seidl R, Birnbacher R, Lischka A, Schober E. Quantitative EEG in young diabetics. J Child Neurol. 1995;10(4): 330-334.

20. Brismar T, Hyllienmark L, Ekberg K, Johansson BL. Loss of temporal lobe beta power in young adults with type 1 diabetes mellitus. Neuroreport. 2002;3(18):2469-2473.

21. Hyllienmark L, Maltez J, Dandenell A. EEG abnormalities with and without relation to severe hypoglycemia in adolescents with type 1 diabetes. Diabetologia. 2005;48:412-419.

22. Cooray G, Hyllienmark L, Brismar T. Decreased cortical connectivity and information flow in type 1 diabetes. Clin Neurophysiol. 2011a;122: 1943-1950.

23. Howorka K, Pumprla J, Saletu B, Anderer P, Krieger M, Schabmann A. Decrease of vigilance assessed by EEG-mapping in type I diabetic patients with history of recurrent severe hypoglycaemia. Psychoneuroendocrinology. 2000;25:85-105.

24. Cooray G, Nilsson E, Wahlin A, Laukka EJ, Brismar K, Brismar T. Effects of intensified metabolic control on CNS function in type 2 diabetes. Psychoneuroendocrinology. 2011b;36:77-86.

25. Tsalikian E, Becker DJ, Crumrine PK, Daneman D, Drash AL. Electroencephalographic changes in diabetic ketosis in children with newly and previously diagnosed insulin-dependent diabetes mellitus. J Pediatr. 1981;99(3):355-359.

26. Haumont D, Dorchy H, Pelc S. EEG abnormalities in diabetic children: influence of hypoglycemia and vascular complications. Clin Pediatr (Phila). 1979;18(12):750-753.

27. Kwon JS, Youn T, Jung HY. Right hemisphere abnormalities in major depression: quantitative electroencephalographic findings before and after treatment. J Affect Disord. 1996;40(3):169-173.

28. Korb AS, Cook IA, Hunter AM, Leuchter AF. Brain electrical source differences between depressed subjects and healthy controls. Brain Topogr. 2008;21(2):138-146.

29. Pizzagalli DA, Oakes TR, Davidson RJ. Coupling of theta activity and glucose metabolism in the human rostral anterior cingulate cortex: an EEG/PET study of normal and depressed subjects. Psychophysiology. 2003;40:939-949.

30. Grin-Yatsenko VA, Baas I, Ponomarev VA, Kropotov JD. Independent component approach to the analysis of EEG recordings at early stages of depressive disorders. Clin Neurophysiol. 2010;121(3):281-289.

31. Pizzagalli DA. Frontocingulate dysfunction in depression: toward biomarkers of treatment response. Neuropsychopharmacology Reviews. 2011;36:183-206.

32. Scheeringa R, Bastiaansen MC, Petersson KM, Oostenveld R, Norris DG, Hagoort P. Frontal theta EEG activity correlates negatively with the default mode network in resting state. Int J Psychophysiol. 2008;67(3):242-251.

33. Buckner RL, Andrews-Hanna JR, Schacter DL. The brain's default network: anatomy, function, and relevance to disease. Ann NY Acad Sci. 2008;1124:1-38.

34. Simpson JR Jr, Snyder AZ, Gusnard DA, Raichle ME. Emotion-induced changes in human medial prefrontal cortex: I. During cognitive task performance. Proc Natl Acad Sci U S A. 2001;98:683-687.

35. Singer W. Synchronisation of cortical activity and its putative role in information processing and learning. Annu Rev Physiol. 1993;55: 349-374. 
36. Muller MM, Gruber T, Keil A. Modulation of induced gamma band activity in the human EEG by attention and visual information processing. Int J Psychophysiol. 2000;38(3):283-299.

37. Mulert C, Pogarell O, Juckel G, et al. The neural basis of the P300 potential. Focus on the time-course of the underlying cortical generators. Eur Arch Psychiatry Clinl Neurosci. 2004;254(3):190-198.

38. Volpe U, Mucci A, Bucci P, Merlotti E, Galderisi S, Maj M. The cortical generators of P3a and P3b: a LORETA study. Brain Res Bull. 2007; 73(4-6):220-230.

39. Cooray GK, Maurex L, Brismar T. Cognitive impairment correlates to low auditory event-related potential amplitudes in type 1 diabetes. Psychoneuroendocrinology. 2008;33:942-950.

40. Kramer L, Fasching P, Madl C, et al. Previous episodes of hypoglycemic coma are not associated with permanent cognitive brain dysfunction in IDDM patients on intensive insulin treatment. Diabetes. 1998;47: 1909-1914.

41. Pozzessere G, Valle E, de Crignis S, et al. Abnormalities of cognitive functions in IDDM revealed by P300 event-related potential analysis. Comparison with short-latency evoked potentials and psychometric tests. Diabetes. 1991;40(8):952-958.

42. Uberall MA, Renner C, Edl S, Parzinger E, Wenzel D. VEP and ERP abnormalities in children and adolescents with prepubertal onset of insulin-dependent diabetes mellitus. Neuropediatrics. 1996;27:88-93.

43. Ziegler D, Hübinger A, Mühlen H, Gries FA. Effects of previous glycaemic control on the onset and magnitude of cognitive dysfunction during hypoglycaemia in type 1 (insulin-dependent) diabetic patients. Diabetologia. 1992;35:828-834.

44. Kurita A, Mochio S, Isogai Y. Changes in auditory P300 event-related potentials and brainstem evoked potentials in diabetes mellitus. Acta Neurol Scand. 1995;92:319-323.

45. Kurita A, Katayama K, Mochio S. Neurophysiological evidence for altered higher brain functions in NIDDM. Diabetes Care. 1996;19(4): 360-364.

46. Tandon OP, Verma A, Ram BK. Cognitive dysfunction in NIDDM: P3 event related evoked potential study. Indian J Physiol Pharmacol. 1999;43(3):383-388.

47. Hegerl U, Gallinat J, Juckel G. Event-related potentials. Do they reflect central serotonergic neurotransmission and do they predict clinical response to serotonin agonists? J Affect Disord. 2001;63(1-2): 93-100.

48. Donald MW, Erdahl DL, Surridge DH, et al. Functional correlates of reduced central conduction velocity in diabetic subjects. Diabetes. 1984;33(7):627-633.

49. Bruder GE, Towey JP, Stewart JW, Friedman D, Tenke C, Quitkin FM. Event-related potentials in depression: influence of task, stimulus hemifield and clinical features on P3 latency. Biol Psychiatry. 1991;30: 233-246.

50. Kawasaki T, Tanaka S, Wang J, Hokama H, Hiramatsu K. Abnormalities of P300 cortical current density in unmedicated depressed patients revealed by LORETA analysis of event-related potentials. Psychiatry Clin Neurosci. 2003;58:68-75.

51. Kemp AH, Hopkinson PJ, Hermens DF, et al. Fronto-temporal alterations within the first $200 \mathrm{~ms}$ during an attentional task distinguish major depression, non-clinical participants with depressed mood and healthy controls: a potential biomarker? Hum Brain Mapp. 2009;30: 602-614.

52. Urretavizcaya M, Moreno I, Benlloch L, et al. Auditory event-related potentials in 50 melancholic patients: increased N100, N200 and P300 latencies and diminished P300 amplitude. J Affect Disord. 2003;74: 293-297.

Neuropsychiatric Disease and Treatment

\section{Publish your work in this journal}

Neuropsychiatric Disease and Treatment is an international, peerreviewed journal of clinical therapeutics and pharmacology focusing on concise rapid reporting of clinical or pre-clinical studies on a range of neuropsychiatric and neurological disorders. This journal is indexed on PubMed Central, the 'PsycINFO' database and CAS.
53. Vandoolaeghe E, van Hunsel F, Nuyten D, Maes M. Auditory event related potentials in major depression: prolonged P300 latency and increased P200 amplitude. J Affect Disord. 1998;48:105-113.

54. Sara G, Gordon E, Kraiuhin C, Coyle S, Howson A, Meares R. The P300 ERP component: an index of cognitive dysfunction in depression? J Affect Disord. 1994;31:29-38.

55. Blackburn IM, Roxborough HM, Muir WJ. Perceptual and physiological dysfunction in depression. Psychol Med. 1990;20:95-103.

56. Gangadhar BN, Ancy J, Janakiramaiah N, Umapathy C. P300 amplitude in non-bipolar, melancholic depression. J Affect Disord. 1993;28: 57-60.

57. Hansenne M, Pitchot W, Gonzalez Moreno A, Zaldua IU, Ansseau M. Suicidal behavior in depressive disorder: an event-related potential study. Biol Psychiatry. 1996;40:116-122.

58. Allison T. Recording and interpreting event related potentials. Cognitive psychophysiology: event-related potentials and the study of human cognition. In: Donchin E, editor. Hillsdale, NJ: Laurence Erlbaum Associates; 1986.

59. Polich J, Kok A. Cognitive and biological determinants of P300: an integrative review. Biol Psychol. 1995;41:103-146.

60. Desmedt JE, Debecker J. Waveform and neural mechanisms of the decision P350 elicited without prestimulus CNV or readiness potential in random sequences of near-threshold auditory clicks and finger stimuli. Electroencephalogr Clin Neurophysiol. 1979;47:648-670.

61. Pritchard WS. Psychophysiology of P300. Psycho Bull. 1981;89: 506-540.

62. Beck AT, Rush AJ, Shaw BF, Emery G. Cognitive therapy of depression. New York. NY: Guilford; 1979.

63. Beck AT, Clark DA. Anxiety and depression: an information processing perspective. Anxietry Res. 1988;1:23-36.

64. Burkhart MA, Thomas DG. Event-related potential measures of attention in moderately depressed subjects. Electroencephalogr Clin Neurophysiol. 1993;88:42-50.

65. el Massioui F, Lesevre N. Attention impairment and psychomotor retardation in depressed patients: an event-related potential study. Electroencephalogr Clin Neurophysiol. 1988;70:46-55.

66. Pfefferbaum A, Wenegrat BG, Ford JM, Roth WT, Kopell BS. Clinical application of the $\mathrm{P} 3$ component of event-related potentials. II Dementia depression and schizophrenia. Electroencephalogr Clin Neurophysiol. 1984;59:104-124.

67. Barry RJ, Johnstone SJ, Clarke AR. A review of electrophysiology in attention-deficit/hyperactivity disorder. II. Event-related potentials. Clin Neurophysiol. 2003;114:184-198.

68. Naatanen R, Picton T. The N1 wave of the human electric and magnetic response to sound: A review and an analysis of the component structure. Psychophysiology. 1987;24:375-425.

69. Rennie CJ, Robinson PA, Wright JJ. Unified neurophysical model of EEG spectra and evoked potentials. Biol Cybern. 2002;86: 457-471.

70. Giard MH, Perrin F, Echallier JF, Thévenet M, Froment JC, Pernier J. Dissociation of temporal and frontal components in the human auditory N1 wave: a scalp current density and dipole model analysis. Electroencephalogr Clin Neurophysiol. 1994;92:238-252.

71. Picton TW, Alain C, Woods DL, et al. Intracerebral sources of human auditory-evoked potentials. Audiol Neurootol. 1999;4:64-79.
The manuscript management system is completely online and includes a very quick and fair peer-review system, which is all easy to use. Visit http://www.dovepress.com/testimonials.php to read real quotes from published authors. 